\title{
La organización informal y el proceso innovador: caso de una PYME colombiana de manufactura electrónica
}

\author{
María Luisa Villalba Morales \\ Docente Universidad de San Buenaventura \\ malu25@gmail.com
}

\author{
Claudia Builes Beltrán \\ Directora General Gemprecol. Colombia. Sur América, Colombia. \\ Chief Executive Officer (CEO) Gemprecol. Colombia South América \\ coordinaciondecampo@genmprecol.com.co
}

(Tipo de Artículo: Investigación Científica y Tecnológica. Recibido el 15/12/2015. Aprobado el 01/04/2016)

\begin{abstract}
Resumen. El objetivo del presente trabajo es evidenciar la importancia de la organización informal como uno de los componentes que influye en el proceso innovador de las pequeñas empresas. Para ello, se analiza el caso de una empresa colombiana de manufactura electrónica, que ha introducido más de 40 productos innovadores al mercado en los últimos 10 años sin contar con una estructura formal para la gestión de la innovación. El referente teórico, tomado como base para el análisis, corresponde al modelo propuesto por López, Díaz, \& Robledo (2013), el cual se sustenta en el modelo conceptual de gestión de la innovación empresarial y los diferentes niveles del comportamiento organizacional. Los resultados obtenidos, permiten resaltar la importancia de la cultura enfocada en la innovación y el liderazgo de la gerencia como los factores de mayor influencia de la organización informal, para el éxito de las innovaciones.
\end{abstract}

Palabras clave. Innovación, Organización informal, Organización formal.

\section{Informal organization and innovative process: case of a colombian electronics manufacturing SME}

\begin{abstract}
The aim of this paper is to show the importance of the informal organization as one of the component that influence the innovative process of SMEs firms. For this reason, the authors analyzed the case of a Colombian company of electronics manufacturing. This firm has introduced more than 40 innovative products to market in the last 10 years without a formal structure for innovation management. The theoretical reference, taken as a basis for analysis, corresponds to that proposed by Lopez, Diaz, \& Robledo (2013), which is based on the conceptual model management business innovation and different levels of organizational behavior model. The results obtained allow to highlight the importance of culture focused on innovation and leadership management as major factors influencing the informal organization, to achieve success of innovations.
\end{abstract}

Keywords. Formal organization, Informal organization, innovation.

\section{Introducción}

En la literatura existe consenso sobre la necesidad de las organizaciones de usar, tanto sus aspectos formales, como sus aspectos informales, para el logro de los objetivos empresariales. Esto gracias a que los primeros aportan a la toma de decisiones deliberadas y orienta el trabajo bajo un diseño organizativo predeterminado [1]; mientras que los segundos corresponde a aquellos acuerdos implícitos que no están escritos, pero influyen significativamente en la forma de actuar de la organización [2, 3].

Al respecto, los escenarios formales e informales capitalizan el desarrollo del recurso humano desde la dirección, la coordinación y la planificación hasta los procesos de adecuación y actuación del grupo modulando la conducta organizacional no solo desde aspectos como la motivación y los estilos de la personalidad, sino además, en la solución de problemas no articulados de manera formal a nivel organizacional pero que si impactan el rendimiento empresarial.

El proceso innovador, a diferencia de la imitación de procesos eficientes de copia y que configuran el modelo convencional, incorporan de forma progresiva habilidades y conocimientos y que habilitan nueva carga de aprendizaje cultural, y que al interior de las organizaciones convergen en tres áreas específicas: estructura, cultura y recursos humanos [4] [5]. En este sentido, es pertinente recabar en la definición de modelos para la medición de las capacidades de innovación, que incluyen el componente formal y el componente informal. Como ejemplo de ello, se tiene el modelo de Nadler \& Tushman [6], el cual busca explicar la organización como un sistema abierto, en el que coexisten diferentes componentes, que entre más alto sea el nivel de congruencia, más eficaz será la organización [6]. De acuerdo a esta formulación, los componentes son: Organización Formal, Sistemas Técnicos, Recursos Humanos y la Organización Informal; sin embargo, a diferencia de la organización formal, la organización informal, como dimensión de la organización, "aún no ha sido suficientemente explorada por quienes han desarrollado investigaciones para evaluar las capacidades de innovación [3, p. 2]".

En este sentido, Farmer [7] ha subrayado la necesidad de explorar el potencial oculto de las redes informales y como desde allí se operacionalizan procesos de comunicación entre gerentes y empleados para aumentar las redes de productividad $y$ externalización de procesos.

En este contexto, llama la atención la existencia de empresas que han tenido un alto desempeño innovador sin contar con una estructura formal para su gestión. Una aproximación a este fenómeno puede explicarse a cambios estructurales en las organizaciones, siendo las empresas con organigramas más horizontales con menor diferenciación vertical y menos estructuras formales, las de mayor posibilidad de innovación por tener estructuras más flexibles. Esta condición puede 
promover la estructura informal como motor principal para el desarrollo de innovaciones exitosas [8]. Una alternativa de análisis de estos casos, corresponde a la utilización del modelo propuesto por Nadler \& Tushman [6] y los niveles del comportamiento organizacional propuestos por Robbins [9], los cuales son: 1) el sistema organizacional, 2) los grupos y 3) las personas.

Por lo tanto, con el fin de evidenciar la relación existente entre la organización informal y el proceso innovador, en este artículo se analiza el primer nivel de la estructura informal [4] de una empresa relacionada a los procesos de ingeniería y manufactura electrónica, como modelo para identificar las fortalezas de dicha estructura y cómo éstas han aportado el éxito de las innovaciones que han tenido.

Con base en lo anterior, a continuación se describe la empresa, seguido del referente teórico usado como base en este trabajo, el cual se compone de la descripción de la estructura formal e informal y los niveles del comportamiento organizacional. Luego se muestra el análisis de cada una de las variables definidas para la comprensión del Sistema Organizacional. Para finalmente presentar las conclusiones obtenidas.

\section{La empresa}

El caso analizado se hizo a una empresa dedicada a la manufactura electrónica, con más de 10 años en el mercado, ubicada en la ciudad de Medellín, Colombia. Actualmente clasifica como empresa pequeña, puesto que cuenta con ocho empleados directos; sin embargo, a este número de empleados se suman asesores técnicos y metodológicos que apoyan el quehacer de la empresa de acuerdo a las particularidades y necesidades de sus proyectos.

El campo específico en el que se desenvuelve la empresa corresponde a la actividad económica: Mantenimiento y reparación especializada de equipo electrónico y óptico, cuyo código ClIU es el 3313. Su portafolio de productos se compone de tres líneas: 1) diseño electrónico; 2) eficiencia y automatización y 3) eficiencia para la cadena de frio. Sus productos se componen de hardware, software y servicio de mantenimiento.

Durante los últimos años han ofrecido al mercado diferentes productos con alto componente tecnológico y usabilidad que favorece el ahorro del consumo de los recursos energéticos y la funcionalidad de equipos industriales a través de la integración con circuitos electrónicos. Para el año 2015 cuenta con 15 productos, distribuidos entre productos a la medida y productos estandarizados, donde dos (2) de ellos han ganado el premio a la innovación (premio ofrecido por entidades promotoras de la innovación a nivel regional). La comercialización de estos dos productos se inició en el año 2014, mismo año en el cual lograron una participación en las ventas del $14.2 \%$.
Lo anterior evidencia el éxito que han tenido sus productos innovadores en el mercado. Sin embargo, el interés por la formalización de la gestión de la innovación en esta organización, surge en los últimos años, y a la fecha han realizado esfuerzos innatos por garantizar nov edad y utilidad en sus productos.

Ante esta situación, y con base en la definición de los compontes del Modelo sistémico de congruencia organizacional de Nadler \& Tushman [6]: organización Formal, personal, tecnología y la organización informal; el proceso innovador que se desarrolla en esta empresa no es el resultado de una organización formal, lo que sustenta la importancia de los otros tres componentes, principalmente la organización informal.

\section{La estructura formal vs la estructura informal}

El proceso innovador, tal como lo ha mostrado la evidencia, puede darse por un proceso estructurado, en el que se plantean y desarrollan estrategias, de forma disciplinada y con objetivos claros [10].

Lo anterior, hace referencia a la organización formal, cuya definición, dada por diferentes autores no genera discrepancias significativas. Por un lado, Burns \& Stalker [11] definen la organización formal, como la estructura mecanicista que fomenta la estandarización de las prácticas de trabajo; mientras que, como componente del modelo de Nadler \& Tushman [6], la organización formal corresponde al conjunto de acciones que orientan el actuar de la organización, tales como las relaciones jerárquicas, existencia de normas, sistemas de comunicación, entre otras [12], es decir, una estructura formal tiende a ser burocrática y se apoya en normas institucionalizadas y políticas empresariales [10].

La importancia de una estructura formal para la innovación, radica en que ésta define la forma en la que interactúan los empleados, facilita la toma de decisiones y se deje menos a la experimentación sin orientación, lo que permite que la capacidad de innovación esté orientada a los objetivos empresariales [10]. Sin embargo, también se ha evidenciado que un alto nivel de formalización puede ir en contra de la creatividad, cualidad inherente en el desarrollo de innovaciones.

En contrapuesta con lo anterior, la estructura informal se define como una estructura organizativa que es orgánica, lo que significa que las normas y las relaciones entre los trabajadores que están menos formalizadas rigen la toma de decisiones y las prácticas de trabajo [10]. En la organización informal se resalta el estilo de gerencia, la cultura organizacional, las relaciones interpersonales e interdepartamentales, los roles y arreglos de trabajo informales y las normas sociales [12].

La importancia de la organización Informal para la innovación, radica en el fomento de una cultura innovadora, en la cual los intereses de los empleados, en la forma como comparten los hábitos y valores, y el trabajo cooperativo para el bien común [3], son mediadores y facilitadores del desarrollo de 
Ing. USBMed, Vol. 7, No. 1, Enero-Julio 2016

innovaciones, pero sin la burocracia de la organización formal.

No obstante, y al igual que la organización formal, la organización informal también puede generar efectos negativos para la organización, y para la innovación, principalmente cuando le vuelve un obstáculo para la creatividad. Lo importante es no desconocer su existencia, para realizar una adecuada gestión de ambos componentes. Los cuales coexisten en las organizaciones y por ello hacen parte del modelo de Nadler \& Tushman [6], como dos de cuatro procesos de transformación (organización formal, sistemas técnicos, recursos humanos y la organización informal), que al mejorar su nivel de congruencia mejorará la eficacia de la organización.

\section{Niveles de comportamiento organizacional de la organización informal}

Robbins [9] propone un modelo, en el cual el comportamiento organizacional, como parte de la organización informal, cuenta con tres niveles jerárquicos, los cuales son: 1) el sistema organizacional, 2) los grupos y 3) las personas. En dichos niveles se agrupa una serie de variables enfocadas a la comprensión y explicación del comportamiento humano en el ámbito del trabajo [3].

Este modelo, junto con el Modelo de Congruencia del Comportamiento Organizacional de Nadler \& Tushman [6], son tomados como referentes teóricos por López, Díaz, \& Robledo [3] para definir, dentro de las jerarquías del comportamiento organizacional, las variables orientadas a la evaluación de las capacidades de innovación de la organización, tal como se muestra en la Tabla 1. Estas variables, específicamente las del nivel: sistema organizacional, son analizadas una a una en la empresa de manufactura electrónica, con el fin de evidenciar el estado de la organización informal en la empresa el efecto positivo que ha tenido en el proceso innovador de la empresa.

\section{SISTEMA ORGANIZACIONAL}

El sistema organizacional, siendo el primer nivel dentro de la jerarquía de los comportamientos organizacionales que representan la organización informal, tienen un comportamiento favorable en la empresa de manufactura electrónica estudiada, el cual se da principalmente por las costumbres y principios propios del personal, al igual que el ambiente propenso a la innovación en el cual están inmersos.

Con base en lo anterior, se analizan las cinco (5) variables del nivel Sistema Organizacional (ver Tabla 1), describiendo cada uno de los aspectos que han garantizado el éxito de sus innovaciones y las bases que pueden facilitar la constitución de una estructura formal para la innovación.

\subsection{Clasificación sectorial}

La empresa pertenece al sector de la manufactura electrónica. El cual es uno de los sectores claves del
Ministerio de las Tecnologías de la Información y la Comunicación de Colombia (Ministerio TIC), gracias a que "La industria electrónica desempeña un papel de primera importancia a nivel mundial por ser un sector de tecnologías estratégicas y transversales" [13, p. 74].

Las perspectivas de crecimiento del sector son alentadoras, tanto para el mercado interno como el internacional. Sin embargo, para el año 2011, Colombia aportó sólo el $0,018 \%$ de las exportaciones mundiales de productos eléctricos y electrónicos [14].

Específicamente, en cuanto a realización de investigaciones básicas en el campo de la electrónica, Colombia presenta un atraso significativo con relación a los países desarrollados, debido al nivel de avance de las capacidades tecnológicas, de formación o económicas, de las empresas [13].

En este sentido, toman relevancia las políticas del estado colombiano definidas para promover el desarrollo del sector en temas de ciencia, tecnología e innovación, lo cual favorece a la empresa, puesto que puede ser potencialmente beneficiaria de los lineamientos gubernamentales que buscan fortalecer el sector a través del fomento de la innovación.

Tabla 1. Variables de la Organización Informal según los niveles de comportamiento [3, p. 13] (1/2)

\begin{tabular}{|c|c|c|}
\hline Nivel & $\begin{array}{l}\text { Nombre de la } \\
\text { variable }\end{array}$ & Descripción de la variable \\
\hline \multirow{5}{*}{ 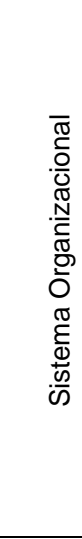 } & $\begin{array}{l}\text { Clasificación } \\
\text { sectorial y } \\
\text { tamaño }\end{array}$ & $\begin{array}{l}\text { Comprende información asociada } \\
\text { al tamaño, sector, activos. }\end{array}$ \\
\hline & $\begin{array}{l}\text { Estrategia } \\
\text { resultados }\end{array}$ & $\begin{array}{l}\text { Lineamiento de orden misional y de } \\
\text { planeación. }\end{array}$ \\
\hline & Tipos de Cultura & $\begin{array}{l}\text { Patrones colectivos der sentir y } \\
\text { actuar, tipificados con instrumentos } \\
\text { como el OCAI. }\end{array}$ \\
\hline & $\begin{array}{l}\text { Aprendizaje } \\
\text { Organizacional }\end{array}$ & $\begin{array}{l}\text { Memoria compartida y la } \\
\text { información externa que impacta } \\
\text { los procesos al interior. }\end{array}$ \\
\hline & Liderazgo & $\begin{array}{l}\text { Características esperadas para } \\
\text { comunicar, administrar, desarrollar } \\
\text { al equipo, relaciones } \\
\text { interpersonales }\end{array}$ \\
\hline
\end{tabular}

Sin embargo, aunque se evidencie un aspecto positivo asociado al sector en el cual la empresa se desenvuelve, el acceso a los recursos y beneficios que ofrece el estado colombiano, requieren de cierto grado de nivel de formalidad, con el cual la empresa aún no cuenta. No obstante, su logro se encuentra planteado como objetivo estratégico, para así mejorar la eficiencia de la Organización. 
Ing. USBMed, Vol. 7, No. 1, Enero-Julio 2016

Tabla 1. Variables de la Organización Informal según los niveles de comportamiento [3, p. 13] (2/2)

\begin{tabular}{|c|c|c|}
\hline Nivel & $\begin{array}{l}\text { Nombre de la } \\
\text { variable }\end{array}$ & Descripción de la variable \\
\hline \multirow{5}{*}{ 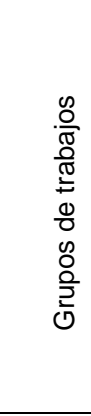 } & Liderazgo & $\begin{array}{l}\text { Características del liderazgo } \\
\text { transformacional en los líderes de la } \\
\text { organización. }\end{array}$ \\
\hline & $\begin{array}{l}\text { Composición del } \\
\text { equipo }\end{array}$ & $\begin{array}{l}\text { Diversidad en atributos de aspectos } \\
\text { visibles y no visibles. }\end{array}$ \\
\hline & Cohesión & Búsqueda de logros conjuntos \\
\hline & Potencia & $\begin{array}{l}\text { Capacidad del equipo para actuar, } \\
\text { hacer cambios, asumir retos. }\end{array}$ \\
\hline & Tareas & $\begin{array}{l}\text { Autonomía que brinda, integración, } \\
\text { oportunidad de aprender. }\end{array}$ \\
\hline \multirow{6}{*}{ 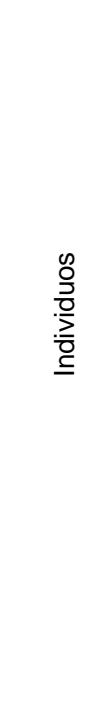 } & $\begin{array}{l}\text { Clima } \\
\text { Crecimiento } \\
\text { Psicológico } \\
\end{array}$ & $\begin{array}{l}\text { Percepción frente a su propio } \\
\text { trabajo. }\end{array}$ \\
\hline & Aprendizaje & $\begin{array}{l}\text { Aparición de nuevas respuestas, } \\
\text { modificación o desaparición de las } \\
\text { actuales. }\end{array}$ \\
\hline & $\begin{array}{l}\text { Crecimiento } \\
\text { Psicológico }\end{array}$ & $\begin{array}{l}\text { Dado por la experiencia y la toma } \\
\text { de conciencia. }\end{array}$ \\
\hline & Compromiso & $\begin{array}{l}\text { Dado por lo cual el trabajador desea } \\
\text { permanecer en la organización. }\end{array}$ \\
\hline & $\begin{array}{l}\text { Autoeficiencia } \\
\text { creativa }\end{array}$ & $\begin{array}{l}\text { Es la creencia que tiene el individuo } \\
\text { y que le lleva a percibir su } \\
\text { capacidad, ante una situación } \\
\text { determinada, para conseguir } \\
\text { resultados en ciertas situaciones. }\end{array}$ \\
\hline & Satisfacción & $\begin{array}{l}\text { Es de orden emocional y está } \\
\text { asociada a la complacencia entre lo } \\
\text { que se esperaba y lo que se } \\
\text { obtiene. }\end{array}$ \\
\hline
\end{tabular}

\subsection{Estrategia y Resultados}

La definición de lineamientos del actuar de la empresa (misión) está soportada en la generación de tecnologías que aporten al progreso de Colombia. Esta orientación está acompañada de un alto nivel de conciencia de la totalidad del personal (técnico, administrativo y directivo) en cuanto a que dicha meta se logra a través de la innovación. Como consecuencia de ello, en los últimos años han lanzado al mercado, productos específicos, cuyos resultados han sido favorables, tanto para la empresa en el marco económico, como para sus clientes en su usabilidad y aporte a la mejora de sus procesos productivos.

A nivel de organización formal, la innovación aún no es explicita en su misión y visión, éstas están soportadas por los pilares: Posicionar; Consolidar y garantiza Rentabilidad. Sin embargo, como meta estratégica, se está construyendo una nueva versión de dicha misión y visión en la cual, la innovación se convierta en unos de los pilares que componen su planeación estratégica.

Con base en lo anterior, se evidencia una coherencia entre la estrategia definida en la empresa, y los resultados alcanzados a la fecha, aunque estos no sean el resultado de una definición explicita de la innovación como pilar para la competitividad, sino un principio implícito de la gerencia y el personal, que cumplen en el día a día.

\subsection{Tipos de Cultura}

Uno de los factores que influye de manera significativa en el desarrollo de innovaciones es la cultura organizacional [15], la cual hace parte de la organización informal y aunque su relación sigue siendo interés de estudio, la mayoría de los artículos empíricos en este campo no utilizan un marco teórico explícito [16] [15].

La importancia del rol de la cultura para la innovación, radica en que ésta influye en los empleados y puede permitir que éstos vean la innovación como un valor fundamental para la empresa y se genere compromiso con ella [16].

Tomando como enfoque los aspectos internos, Abdullah et al. [17], muestran la relación positiva entre la cultura organizacional y la innovación de productos de las pymes de Malaysia. El enfoque de este trabajo se hizo a través de cuatro aspectos importantes de la cultura organizacional: la misión, la consistencia, la adaptabilidad y la participación de los empleados. Ante ello se concluye sobre la importancia de: 1) incluir la innovación en la misión y la visión; 2) garantizar el compromiso y 3) promover la participación de los empleados en las actividades de innovación.

En concordancia con este trabajo, la empresa de manufactura electrónica estudiada se caracteriza por tener una cultura organizacional fuerte, enfocada a la innovación. Puesto que existe un alto compromiso por parte de los empleados con la empresa, la generación de soluciones y la satisfacción de los clientes. De igual forma, el nivel de participación en los proyectos de innovación por parte de los empleados es significativo. Más del $60 \%$ de los empleados ha participado en los proyectos, desde la concepción de la idea, la formulación, hasta la ejecución y cierre de los mismos.

Los roles de cada uno de los empleados está totalmente claro, principalmente cómo su trabajo aporta al logro de los objetivos empresariales y cómo sus actividades aportan al proceso innovador, aunque no cuentan con una dependencia o responsable directo del sistema de innovación.

\subsection{Aprendizaje Organizacional}

Entendiéndose el aprendizaje organizacional como el proceso por el cual las organizaciones aprenden y desarrollan nuevos conocimientos [18], en la empresa se pueden encontrar evidencias del desarrollo de los subprocesos que hacen parte del proceso del aprendizaje organizacional [18], lo que ha permitido la creación de ese "repositorio" de información histórica que "posibilita el desarrollo de capacidades de innovación" [3, p. 8], tal como se muestra en la Tabla 2.

\subsection{Liderazgo}

Según Oke, Munshi, \& Walumbwa [19], el liderazgo es visto como un proceso social que toma lugar en un 
Ing. USBMed, Vol. 7, No. 1, Enero-Julio 2016

contexto grupal donde el líder influye sobre sus seguidores para el logro de las metas conocidas. Por lo tanto, el rol del líder debe ser inspirador motivador y visionario, de tal modo que se llegue a un diseño apropiado de contexto organizacional. Con base en ello, el Gerente de la empresa de manufactura electrónica, se caracteriza por identificar las oportunidades de cambios en el futuro, lo que le permite preparar, tanto al personal, como la infraestructura tecnológica de la empresa para los próximos cambios de tendencias, tanto de clientes como de tecnologías.

Tabla 2. Acciones de aprendizaje organizacional en la empresa

\begin{tabular}{|c|c|}
\hline Subproceso & Evidencia \\
\hline \multirow{4}{*}{$\begin{array}{l}\text { Adquisición de } \\
\text { conocimiento }\end{array}$} & $\begin{array}{l}\text { No existe un proceso definido para la adquisición } \\
\text { de conocimiento. }\end{array}$ \\
\hline & $\begin{array}{l}\text { Asistencia a capacitaciones en temas de } \\
\text { manufactura electrónica, gestión de la innovación } \\
\text { y elaboración de proyectos. }\end{array}$ \\
\hline & $\begin{array}{l}\text { Realización de vigilancias tecnológicas de corto } \\
\text { alcance }\end{array}$ \\
\hline & $\begin{array}{l}\text { Participación de expertos en el desarrollo de } \\
\text { proyectos }\end{array}$ \\
\hline Distribución & $\begin{array}{l}\text { De forma intuitiva la empresa distribuye el } \\
\text { conocimiento a través de las siguientes } \\
\text { herramientas: 1) uso de actas de reunión y correo } \\
\text { electrónico, 2) realización de reuniones periódicas } \\
\text { para el análisis de los problemas de los clientes y } \\
\text { sus posibles soluciones, y 3) generación de fichas } \\
\text { de desarrollo de productos, manuales, planos y } \\
\text { diseños esquemáticos de producto. }\end{array}$ \\
\hline Interpretación & $\begin{array}{l}\text { Existe un equipo técnico encargado de analizar y } \\
\text { discutir los conocimientos adquiridos, con el fin de } \\
\text { definir las soluciones de mayor impacto para los } \\
\text { problemas de los clientes. Este análisis permite la } \\
\text { identificación de oportunidades de innovación y la } \\
\text { definición de los futuros proyectos. }\end{array}$ \\
\hline Memoria & $\begin{array}{l}\text { Con base en el desarrollo de las anteriores etapas, } \\
\text { la empresa construye su "repositorio" de } \\
\text { información histórica que contribuye al desarrollo } \\
\text { de las capacidades de innovación (López, Díaz, \& } \\
\text { Robledo, 2013) y a evitar la pérdida de } \\
\text { conocimiento debido a la rotación del personal. } \\
\text { Actualmente se puede acceder a investigaciones } \\
\text { previas, fichas de desarrollo de productos, } \\
\text { manuales, planos y diseños esquemáticos de } \\
\text { producto. }\end{array}$ \\
\hline
\end{tabular}

De igual manera, se ha encargado fielmente de facilitar la comunicación, no sólo de forma horizontal, sino también con la gerencia. En la empresa, la comunicación se caracteriza por ser bilateral, en donde se reconoce el valor de la opinión y las ideas. Por otro lado, el equipo de trabajo cuenta con posibilidades de mejoramiento profesional y personal, a través de capacitaciones y/o interacción directa con los clientes, donde la participación siempre el lideradas y motivas desde la gerencia. Tal como lo dice uno de los entrevistados: "La gerencia no es un departamento lejano, intenta satisfacer los deseos de los empleados y de los clientes".

En cuanto a las relaciones interpersonales, se cuenta con seis (6) principios, que nutren el valor moral de los empleados, estos son Responsabilidad, la Honestidad, el Respeto, la Solidaridad, el compromiso y la Fe. Cada empleado tiene estos principios claros y los aplica tanto para los objetivos empresariales, como para la definición de las innovaciones.

\section{Conclusiones}

Las evidencias encontradas en la empresa de manufactura electrónica estudiada, en cuanto a las fortalezas que presenta en su organización informal, específicamente en el nivel Sistema organizacional, ratifican la importancia de fomentar esta estructura para desarrollar capacidades de innovación.

Cada una de las cinco (5) variables analizadas aporta al éxito de la innovación, destacándose aquellas que promueven el entendimiento y asociación del personal con el concepto e importancia de la innovación, como requisito fundamental para que la organización sea eficiente. Estas variables son la cultura enfocada a la innovación y el liderazgo de la gerencia. Puesto que estas dos variables han permitido que en la empresa se genere un proceso de aprendizaje y de definan estrategias claras y se obtengan resultados favorables.

En este sentido, la empresa evidencia la disposición al cambio, dando un paso significativo en la gestión de la innovación, al contar con una estructura informal favorable. Ahora, para dar el siguiente paso, definir e implementar la estructura formal, se debe hacer con el propósito de aumentar el nivel de congruencia entre los diferentes componentes (estructura formal, estructura informal, personal y tecnología) para garantizar un mayor nivel de eficacia, tal como se sustenta en el modelo de Nadler \& Tushman [6].

\section{Agradecimientos}

Las autoras agradecen a cada uno de los empleados de la empresa de manufactura electrónica ÓMICRON INGENIERÍA S.A.S, por su participación en el desarrollo de esta investigación.

\section{Referencias}

[1] O. Rank, "Formal structures and informal networks: Structural analysis in organizations," Scandinavia Journal of Management, no. 24, p. 145-161, 2008.

[2] F. Angeli, "With the help of a foreign ally: biopharmaceutical innovation in India after TRIPS," Health Policy Plannig, vol. 29, no. 3, pp. 280-291, 2014.

[3] C. López, P. Díaz and J. Robledo, "La Organización Informal y sus Efectos en las Capacidades de Innovación," in XV Congreso Latino- Inberoamericano de Gestión Tecnológica. Las nuevas condiciones y espaciona para el desarrollo científico, tecnológico e industrial y la cooperación international, Porto, 2013.

[4] C. H. Legare and M. Nielsen, "Imitation and Innovation: The Dual Engines of Cultural Learning.," Trends in Cognitive Sciences, vol. 19, no. 11, pp. 688-699, 2015.

[5] C. H. Legare, N. J. Wen, P. A. Herrmann and H. Whitehouse, "Imitative flexibility and the development of cultural learning," Cognition, vol. 142, p. 351-361, 2015. 
Ing. USBMed, Vol. 7, No. 1, Enero-Julio 2016

[6] D. A. Nadler and M. L. Tushman, "Congruence Model for Organization Problem Solving," in Managing Satrategic Innovation and Change, Oxford University Press, 1997, p. 159171.

[7] N. Farmer, The invisible organization : how informal networks can lead organizational change, Farnham: Gower, 2008.

[8] C. Rodgers, Informal coalitions : mastering the hidden dynamics of organizational change, Basingstoke: Palgrave Macmillan, 2007.

[9] S. Robbins, Comportamiento Organizacional, 10 ed., México: Pearson Education, 2004.

[10] B. Menguc and S. Auh, "Development and return on execution of product innovation capabilities: The role of organizational structure," Industrial Marketing Management, vol. 39, p. 820831, 2010.

[11] T. Burns and G. M. Stalker, The management of innovation., London: Tavistock, 1961.

[12] J. Robledo, C. López, W. Zapata and J. D. Pérez, "Desarrollo de una Metodología de Evaluación de Capacidades de Innovación," Perfil de Coyuntura Económica, no. 15, pp. 133148, 2010.

[13] C. Ministerio TIC, "Plan Nacional de Ciencia, Tecnología e Innovación para el desarrollo de los sectores ELECTRÓNICA,
TECNOLOGIAS DE LA INFORMACIÓN Y LAS COMUNICACIONES (ETIC)," 2014. [Online]. Available: http://www.fiti.gov.co/lmages/Recursos/5_Plan_Nacional_de_ CTI.pdf. [Accessed 2210 2014].

[14] DANE, "Exportaciones Colombianas de productos electrónicos 2002-2010," 2011.

[15] A. Turró, D. Urbano and M. Peris, "Culture and innovation: The moderating effect of cultural values on corporate entrepreneurship," Technological Forecasting \& Social Change, no. 88, p. 360-369, 2014.

[16] J. C. Naranjo Valencia, D. Jiménez Jiménez and R. Sanz Valle, "¿Es la cultura organizativa un determinante de la innovación en la empresa?," Cuadernos de Economía y Dirección de la Empresa, vol. 15, p. 63-72, 2012.

[17] N. H. Abdullah, A. Shamsuddin, E. Wahab and N. A. Abdul Hamid, "The Relationship between Organizational Culture and Product Innovativeness," Procedia - Social and Behavioral Sciences, vol. 129, p. $140-147,2014$.

[18] M. I. Barba, D. Jiménez and R. Sanz, "Training and performance: The mediating role of organizational learning," BRQ Business Research Quarterly, vol. 17, pp. 161 - 173, 2014.

[19] D. Oke, N. Munshi and F. O. Walumbwa, "The Influence of Leadership on Innovation Processes and Activities," Organizational Dynamics, vol. 38, no. 1, pp. 64-72, 2009. 